Rev. Biol. Neotrop. 9(1): 10-18. 2012

\title{
ORFOLOGIA DE FITÓLITOS CARACTERÍSTICOS DE DUAS ESPÉCIES DE Arecaceae do bioma Mata Atlântica: Bactris setosa Mart. e Geonoma schottiana Mart.
}

\author{
Mayara dos Reis Monteiro \\ Laboratório de Estudos Paleambientais da Felcicam. Avenida Comendador Norberto Marcon- \\ des, 733, 87303-100, Campo Mourão, Paraná, Brasil; e-mail: mayarareismonteiro@gmail. \\ com.
}

\section{Janaina Silva Rossi Pereira}

Laboratório de Estudos Paleambientais da Felcicam. Avenida Comendador Norberto Marcondes, 733, 87303-100, Campo Mourão, Paraná, Brasil; e-mail: j_janaina_a@hotmail.com

\section{Giliane Gessica Rasbold}

Laboratório de Estudos Paleambientais da Felcicam. Avenida Comendador Norberto Marcondes, 733, 87303-100, Campo Mourão, Paraná, Brasil; e-mail: grasbold@gmail.com.

\section{Mauro Parolin}

Laboratório de Estudos Paleambientais da Felcicam. Avenida Comendador Norberto Marcondes, 733, 87303-100, Campo Mourão, Paraná, Brasil; e-mail: mauroparolin@gmail.com.

\section{Marcelo Galeazzi Caxambu}

Herbário HCF, Universidade Tecnológica Federal do Paraná. BR 369, Km 0,5, 87301-006, Campo Mourão, Paraná, Brasil.

\begin{abstract}
Resumo: Foi realizada a caracterização e quantificação das morfologias de fitólitos (corpos micrométricos de opala biogênica) da bráctea peduncular, dos folíolos e da ráquila de Bactris setosa Mart. e Geonoma schottiana Mart. (Arecaceae que têm ocorrência na Mata Atlântica). A extração foi realizada via dissolução ácida, posteriormente os fitólitos foram contados (400 grãos por lâmina, três lâminas por estrutura vegetal), foi realizada a medição de seus diâmetros e corte histológico dos folíolos. $B$. setosa apresentou de maneira predominante (>98\% de ocorrência) o fitólito "conical echinate", com diâmetros

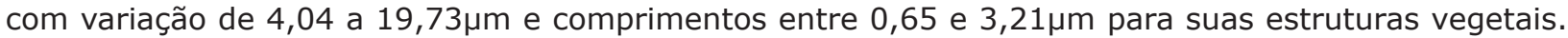
Tal morfologia apresenta-se disposta alinhada longitudinalmente no folíolo. Em G. schottiana, o fitólito predominante é o tipo "globular echinate" (>98,5\%), com diâmetros A que variam de 4,41 a 13,07um e

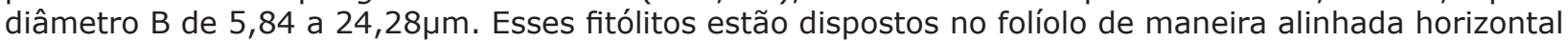
e transversalmente.
\end{abstract}

Palavras-chave: Conical echimate, Globular echimate, Opala silicosa.

Aвstract: We performed the characterization and quantification of the morphology of phytoliths (biogenic opal micrometric bodies) of bract stalk, leaflets and rachilla of Bactris setosa Mart and Geonoma schottiana Mart. (Arecaceae that has occurred in the Mata Atlantica). The extraction was carried out by acid dissolution, and then the phytoliths were counted (400 grains per slide, three slides per plant structure), their diameters were measured and the histological section of the leaflets was performed. $B$. setosa showed predominately ( $>98 \%$ occurrence) the conical echinate phytolith, they have diameters ranging between 4.04 to $19.73 \mu \mathrm{m}$ and lengths from 0.65 to $3.21 \mu \mathrm{m}$ in this plant structure, such morphology appears aligned along the leaflet. In G. schottiana, the predominant phytolith is the Globular echinate ( $>98.5 \%$ ) with A diameters ranging from 4.41 to $13.07 \mu \mathrm{m}$ and B diameters ranging from 5.84 to $24.28 \mu \mathrm{m}$. These phytoliths are arranged horizontally and transversely aligned on the leaflet.

KeY wORDS: Biogenic opal, Conical echimate, Globular echimate. 


\section{INTRODUÇÃO}

De acordo com Piperno (1988), fitólitos são corpos micrométricos de opala silicosa precipitados nos tecidos ao longo da vida de um vegetal. Segundo Madella (2008), ao retirar água e nutrientes do solo as plantas absorvem o ácido silícico $\mathrm{Si}\left(\mathrm{OH}_{4}\right)$. Os íons de $\mathrm{Si} \mathrm{são}$ então induzidos a precipitar e cristalizar em células moldes criadas pelo organismo vegetal. Esse processo é conhecido por Osterrieth (2008) como biomineralização e pode ocorrer em diversas partes das plantas.

A biomineralização da sílica nos vegetais pode ocorrer em folhas, sementes, frutos, raízes e madeira; dentro das células ou na parede celular (Sendulsky \& Labouriau, 1966; Runge, 1999). Quando o vegetal, ou parte dele morre, os fitólitos podem ficar acumulados no solo, o que têm lhes conferido o título de registros fósseis terrestres mais duráveis e por isso constituem uma importante ferramenta de interpretação nos estudos paleoambientais e arqueológicos.

O conhecimento sobre os fitólitos remontam ao século XIX, conforme expressa Zucol et al. (1999), em sua extensa lista de referencias sobre o tema. Devido a sua resistência à decomposição, os fitólitos são muito utilizados em trabalhos de reconstrução paleoambiental e em arqueologia. Nesse sentido esses corpos silicosos assumem uma importância impar, pois podem ser usados em locais onde, por exemplo, a preservação polínica não é favorecida.

No Brasil, não são muitos os trabalhos de descrição morfológica de fitólitos podendo ser citados as pesquisas pioneiras de Sendulsky e Laboriau (1966); Cavalcante (1968); Campos e Laboriau (1969), e, mais recentemente os de Medeanic et al (2008); Rasbold et al (2011) e Raitz (2012). Com relação ao emprego desses corpos silicosos em estudos paleoambientais, pode-se citar mais recentemente para o Brasil o trabalho de Coe et al. (2012) e Monteiro et al (2011).

Dessa forma, a quantidade e a morfologia dos fitólitos encontrados em plantas atuais é um referencial para a identificação taxonômica em sedimentos (Família e por vezes Gênero). Tal fato evidencia a necessidade de pesquisas com plantas atuais para a correta calibração dos estudos paleoambientais.
A família Arecaceae tem aproximadamente 240 gêneros e 2600 espécies, sendo 37 gêneros e 208 espécies nativas no Brasil (Lorenzi 1996). Na Mata Atlântica ocorrem cerca de 40 espécies de palmeiras, pertencentes a 10 gêneros (Henderson et al., 1995) dentre eles Bactris sp. e Geonoma sp., em que se enquadram as duas espécies alvo deste trabalho, Geonoma schottiana Mart. e Bactris setosa Mart.

Bactris setosa é uma espécie cespitosa, que forma touceiras densas de até $6 \mathrm{~m}$ de altura com diâmetro de $3-4 \mathrm{~cm}$. O caule e as pinas possuem espinhos curtos de cor palha com a extremidade preta, distribuídos uniformemente na face abaxial da bainha, pecíolo e raque. Os frutos são globosos, roxo-escuros quando maduros, com polpa suculenta. Sua frutificação é no verão e as sementes têm germinação lenta e baixa. Ocorre desde o sul da Bahia até o Rio Grande do Sul na costa litorânea, no sub-bosque da floresta Atlântica, bem como na vegetação secundária e áreas abertas, principalmente em solos úmidos e brejosos (Lorenzi et al, 2010).

Geonoma schottiana é uma palmeira monóica, de caule solitário que eventualmente forma touceiras, tolerante a sombra e de crescimento lento, chega a $4 \mathrm{~m}$ de altura ocupando o sub-bosque da floresta. Suas folhas são divididas em numerosas pinas estreitas falciformes e seus frutos são do tipo ovóide (globosos), ficando maduros entre março e setembro. A espécie possui alta produtividade de frutos sendo dispersos por aves (Lorenzi et al, 2010). Ocorre de Santa Catarina a Goiás, nas florestas costeiras planas ou florestas de encosta, restingas e capoeirões.

Dada à importância ecológica da família Arecaceae, o seguinte trabalho visa apresentar os morfotipos de fitólitos característicos das espécies B. setosa e G. schottiana, a fim de auxiliar estudos paleoambientais.

\section{Material e mÉtodos}

A coleta do espécime de $B$. setosa foi realizada em março de 2012 na região de Porto Rico, Paraná. Já o espécime G. schottiana foi coletado em novembro de 2011, no Buraco do Padre em Ponta Grossa, Paraná. Os espécimes foram identificados no Herbário da UTFPR 
(HCF), tendo como esteio, respectivamente, os exemplares HCF 10.800 e HCF 63.340.

Para a obtenção dos fitólitos das palmeiras separou-se $3 \mathrm{~g}$ de material, da bráctea peduncular, dos folíolos e da ráquila (Fig. 1), posteriormente lavado e seco em estufa $\left(60^{\circ} \mathrm{C}\right)$. As amostras foram acomodadas em beckers e encaminhadas para tratamento químico (adaptado de Medeanic et al., 2008), visando a dissolução do material orgânico e celulose. Neste tratamento, as porções receberam solução de
1:4 de ácido nítrico $\mathrm{HNO}_{3}(65 \%)$ e ácido sulfúri$\mathrm{co} \mathrm{H}_{2} \mathrm{SO}_{4}$ respectivamente, sendo aquecidas em chapa térmica a $90^{\circ} \mathrm{C}$ por um período de três horas. Ao final da etapa de queima química, cada alíquota recebeu aproximadamente $10 \mathrm{ml}$ de peróxido de hidrogênio $\mathrm{H}_{2} \mathrm{O}_{2}$ (v.130). Com objetivo de neutralizar as amostras, foram realizadas lavagens com água destilada, até que o pH atingisse valores básicos, agilizando-se o processo via centrifugação (1.500 rpm/3min).

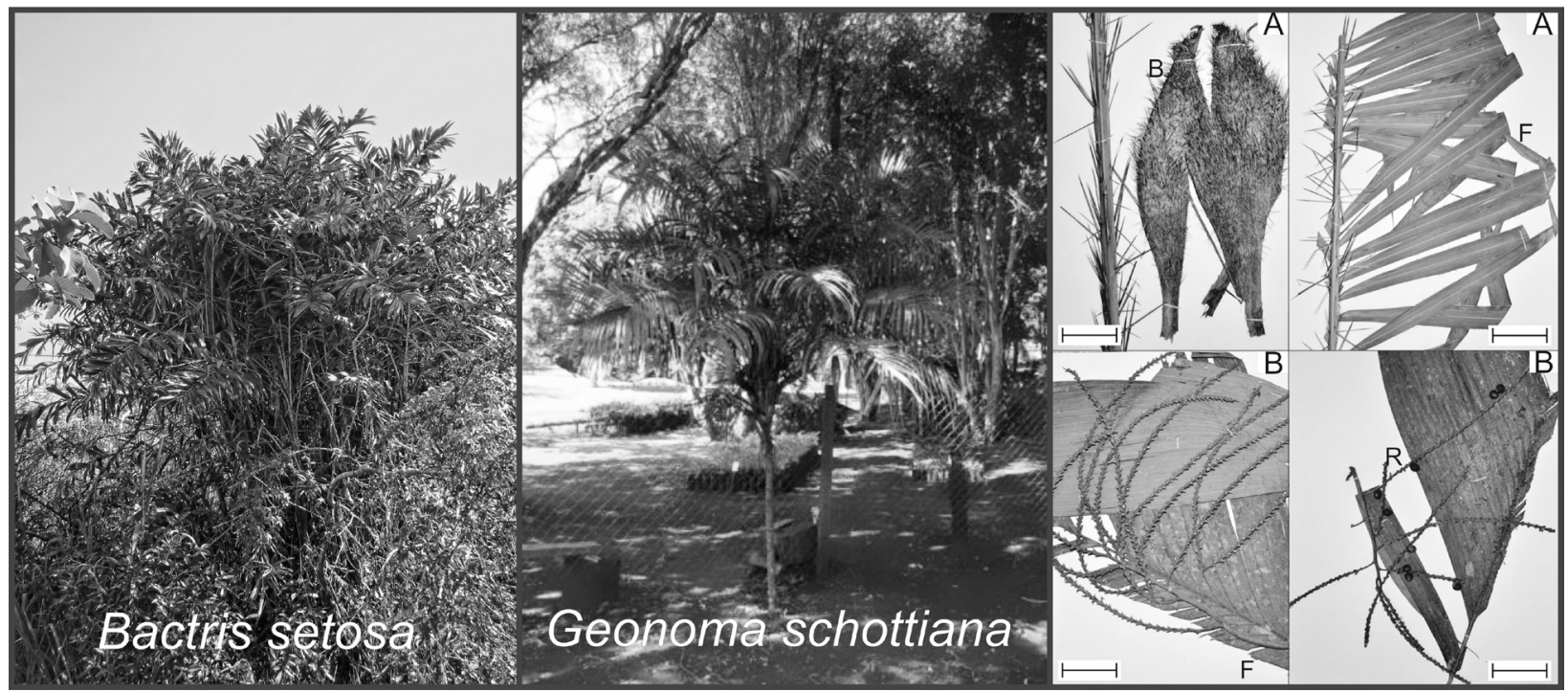

Figura 1. Espécies Bactris setosa e Geonoma schottina em seu habitat. Estruturas vegetais analisadas A, Bactris setosa, B. Geonoma schottiana.; R. Ráquila, F. Folíolo, B. Bráctea - escala 4cm.

As lâminas foram montadas com $50 \mu \mathrm{L}$ de material processado, secas em chapa térmica e cobertas com Entellan® e lamínula. As identificações, observações e microfotografias foram realizadas em microscópio óptico com aumento de 40x.

Para a contagem foram determinados quatrocentos grãos por lâmina, 3 lâminas por estrutura vegetal. Posteriormente foi realizada a análise cluster de similaridade euclidiana (software Past ${ }^{\circledR}$ ), e a medição de $10 \%$ de cada morfologia representativa em cada estrutura vegetal, através do software TSView $7^{\circledR}$.

Para a determinação da posição dos fitólitos no folíolo do espécime, foi utilizada a técnica de clareamento por remoção de celulose e outros compostos orgânicos, que consiste no repouso de um pequeno fragmento da parte analisada (corte longitudinal do folíolo, $\sim 0,5 \mathrm{~cm}$ ) em uma solução de 1:1 de hipoclorito de sódio $(\mathrm{NaClO})$ e água destilada, por um período de 24 horas. Após clareamento, a amostra vegetal foi colocada em lâmina, seca em chapa térmica e fixada com lamínula e Balsamo do Canadá ${ }^{\circledR}$.

A identificação morfológica foi realizada através da comparação com os trabalhos de Madella et al (2005), Piperno (2006), Medeanic et al (2007; 2008), Lu et al. (2007), Zucol \& Brea (2005) e coleção de referencia de fitólitos do Laboratório de Estudos Paleoambientais da Fecilcam (Lepafe). A terminologia utilizada foi a proposta no International Code for Phytolith Nomenclature 1.0, (Madella et al 2005). Para evitar problemas de tradução optou-se por manter as terminologias em língua inglesa.

As lâminas analisadas estão depositadas no Lepafe sob os códigos L.175, C.16 e L.177, C.17.

\section{Resultados e discussão}

A análise de B. setosa e G. schottiana evidenciou a existência de respectivamente, 8 e 7 morfologias de fitólitos para cada espécie (Tab. 1). 
Tabela 1 - Morfotipo de fitólitos e sua quantidade nas diferentes estruturas de G. schottiana e B. setosa

\begin{tabular}{lcccccc}
\hline \multirow{2}{*}{ Morfotipo de Fitólito } & \multicolumn{3}{c}{ Geonoma schottiana } & \multicolumn{3}{c}{ Bactris setosa } \\
& Bráctea & Folíolo & Ráquila & Bráctea & Folíolo & Ráquila \\
\hline Bilobate & 0 & 0 & 3 & 3 & 1 & 1 \\
Conical echinate & 0 & 0 & 0 & 1176 & 1190 & 1086 \\
Cylindric sulcate tracheid & 7 & 2 & 0 & 5 & 0 & 91 \\
Globular echinate & 1182 & 1184 & 1184 & 0 & 0 & 0 \\
Elongate dendriform & 0 & 0 & 0 & 0 & 1 & 0 \\
Papilae & 0 & 0 & 2 & 9 & 3 & 4 \\
Elongate psilate & 11 & 10 & 8 & 6 & 3 & 4 \\
Rondel & 0 & 0 & 0 & 0 & 1 & 0 \\
Sadle & 0 & 3 & 3 & 1 & 0 & 0 \\
Trapeziform polylobate & 0 & 1 & 0 & 0 & 0 & 0 \\
\hline
\end{tabular}

O morfotipo "globular echinate" foi encontrado, em grande quantidade, em todas as partes analisadas de G. schottiana, evidenciando sua representatividade como morfotipo característico da espécie (Fig. 2). Tal morfologia é descrita por Zucol \& Brea (2005) como fitólito esférico com ornamentações espinhosas, tendo diâmetro variável de 5-35 $\mu$ m, tal como acontece em G. schottiana (Fig. 3). Na espécie estudada, a morfologia "globular echinate" apresen-

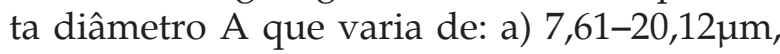

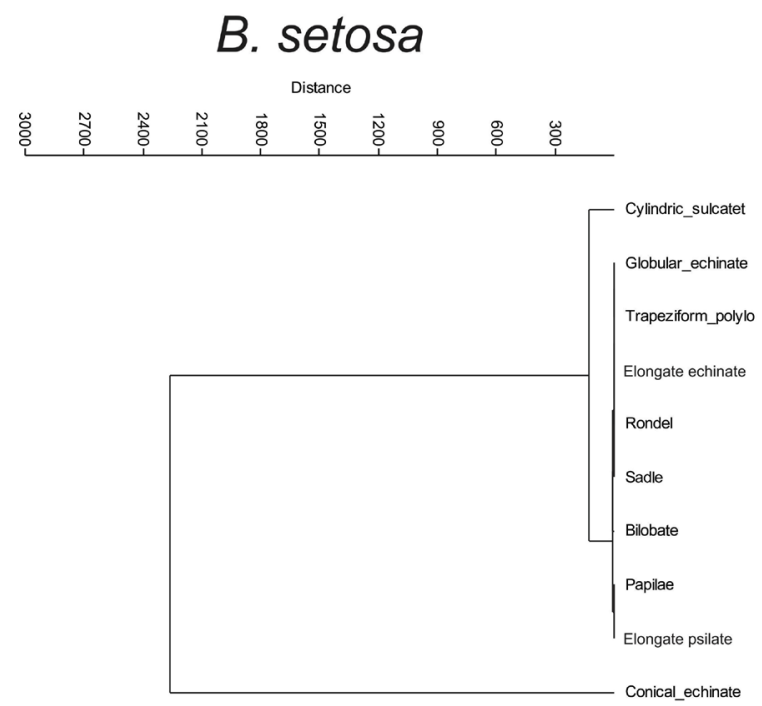

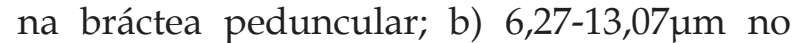

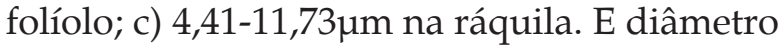

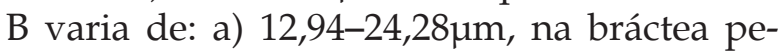
duncular; b) 10,94-19,32 um no folíolo; c) 5,84$13,40 \mu \mathrm{m}$ na ráquila. Esta forma foi encontrada ainda no Butia capitata (Mart.) Becc. em Piperno (2006) e no Butia paraguayensis (Barb. Rodr.) L. H. Bailey em Rasbold et al.(2011), que a qualificaram como nitidamente característico para este gênero da família Arecaceae.

\section{G. schottiana}

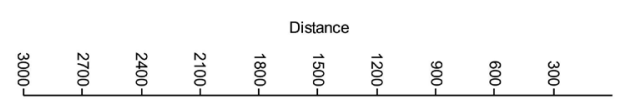

Figura 2. Análise cluster de similaridade euclidiana.

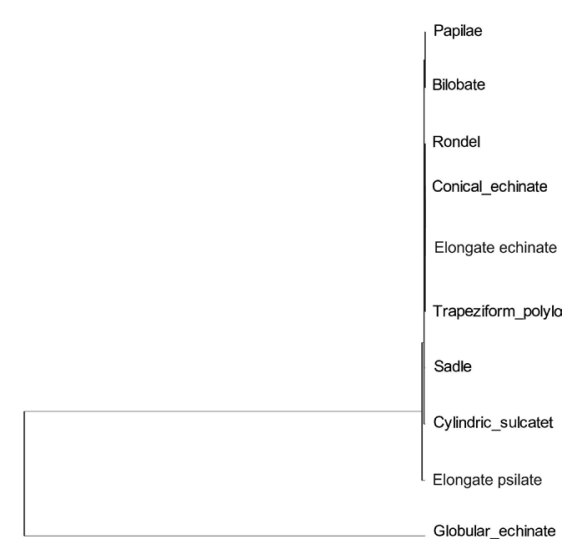




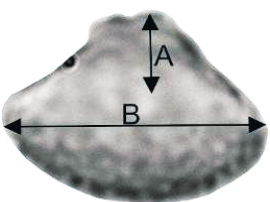

Conical echinate

B. setosa

2,20

1,03

1,47

19,73

4,52

8,52

3,21

0,81

1,93

20,19

4,04

6,98

2,86

0,65

1,65

14,71

4,61

8,66

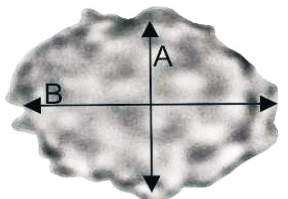

Globular echinate

G.schottiana

20,12

7,61

13,67

24,28

12,94

17,92

13,07

6,27

10,11

19,32

10,94

15,00

11,73

4,41

7,44

13,40

5,84

10,83

Figura 3. Dimensões dos fitólitos "conical echinate" e "globular echinate" nas espécies B. setosa e G. schottiana, respectivamente.

A espécie B. setosa apresentou como morfotipo característico a forma "conical echinate" (Fig. 2), assim denominada por sua estrutura cônica (larga e robusta na base afilando em direção ao ápice) com ornamentações espinhosas em toda a forma (Fig. 4). Tal morfologia já foi descrita para as espécies Geonoma interrupta (Ruiz \&Pavon) Martius e Caryota rumphiana Mart. (Piperno,1988), e Caryota mitis Lour. (Kealhofer \& Piperno. 1998), no entanto, estes levantamentos não fazem menção as dimensões da estrutura fitolítica. Na espécie $B$. setosa a morfologia "conical echinate" apresentou comprimentos
(A) (Fig. 3) que variam de: a) 1,03-2,20 $\mu \mathrm{m}$, na bráctea peduncular; b) 0,81-3,21 $\mu \mathrm{m}$ no folíolo; c) 0,65-2,86um na ráquila; e diâmetros

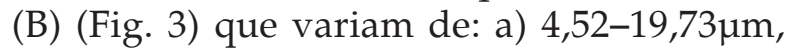

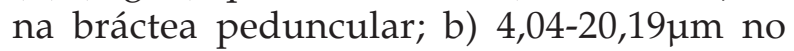

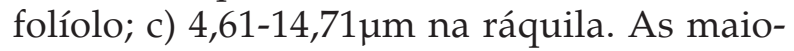
res médias de diâmetro foram registradas na bráctea peduncular para o G. schottiana, e na ráquila para o $B$. setosa, e as menores na ráquila (G. schottiana) e no folíolo (B. setosa). Quanto à média de comprimento da morfologia "conical echinate" na espécie $B$. setosa, o maior valor ocorreu na bráctea peduncular, e o menor no folíolo. 


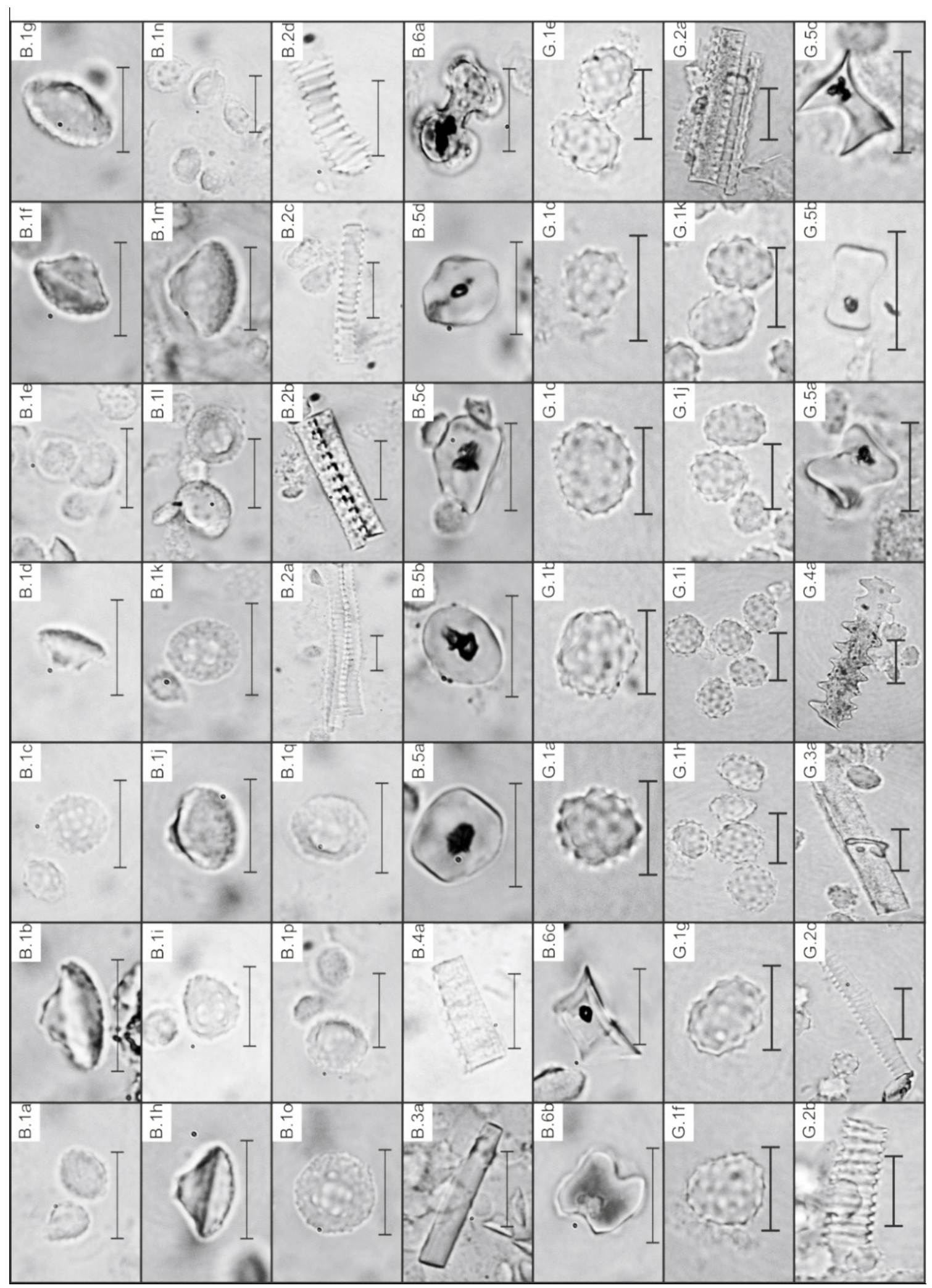

Figura 4. B: B. setosa, G: G. schottiana; B1a - B1. "conical echinate", B2a - B2d. "cylindric sulcate tracheid", B3a. "elongate psilate", B4a. "elongate dendriform", B5a - B5d. "papillae", B6a - B6c. "bilobate", G1a - G1k. "globular echinate", G2a

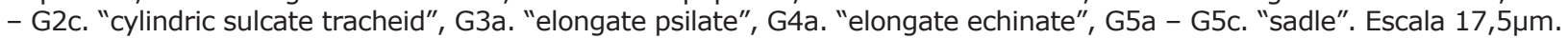


Outras formas fitolíticas, além das representativas para cada espécie, compõe menos de $2 \%$ das morfologias encontradas, o que correlacionado a análise cluster, que evidenciou a maior similaridade entre essas outras morfologias e uma discrepância das morfologias "globular echinate" e "conical echinate" em relação ao grupo, evidencia a pouca representatividade das outras formas de fitólitos para a assembleia das espécies estudadas (Tab.1, Fig.2).
A localização dos fitólitos "globular echinate" e "conical echinate" no folíolo das espécies G. schottiana e B. setosa, respectivamente, foi demonstrada através do corte histológico (Fig. 5), evidenciando maior simetria na disposição dos fitólitos na espécie G. schottiana. Na espécie $B$. setosa observa-se um alinhamento longitudinal dos fitólitos, enquanto, na espécie G. schottiana esse alinhamento também é observado no sentido transversal.

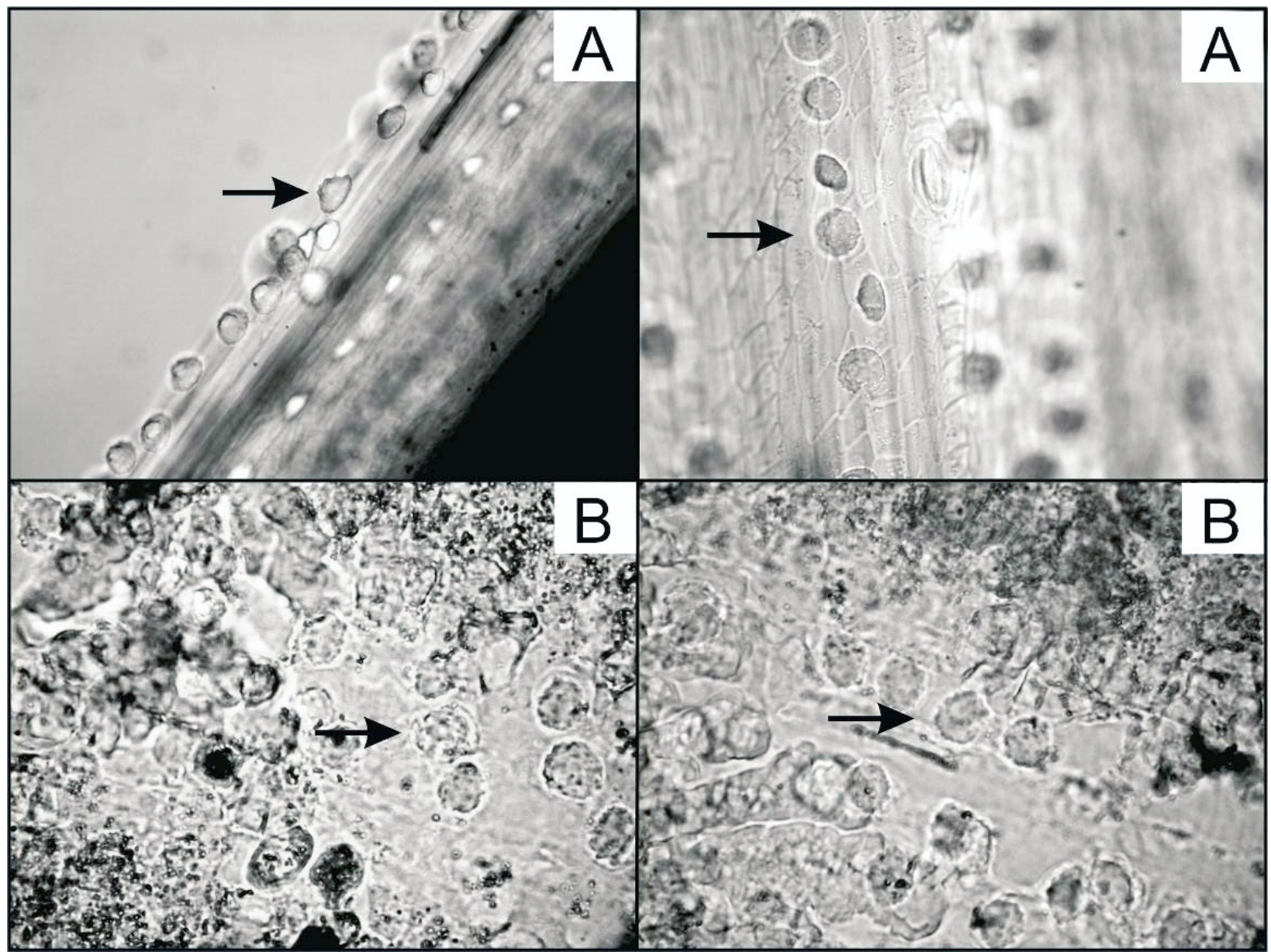

Figura 5. Corte longitudinal do folíolo: A- B. setosa; B- G. schottiana. Seta: 17,5 $\mu \mathrm{m}$.

\section{Conclusão}

A morfologia representativa das espécies $G$. schottiana e B. setosa, como visto através da análise cluster, é, respectivamente, "globular echinate" e "conical echinate", as quais ocorrem em grande quantidade nas três estruturas vegetais analisadas.

Para futuras reconstruções paleoambientais estima-se que as espécies estudadas possam contribuir de maneira significativa, tendo em vista a grande produção de fitólito representativo ("globular echinate" e "conical echinate") apresentada pelas duas palmeiras típicas do bioma Mata Atlântica.

\section{Agradecimentos}

A primeira e a terceira autoras agradecem ao processo do CNPq $n^{\circ} 552980 / 2011-0$, pela concessão de bolsas de apoio técnico. $\mathrm{O}$ quarto autor agradece ao $\mathrm{CNPq}$ (processos 400442/2010-8 e 401765/2010-5). 


\section{REFERÊNCIAS}

Campos, A.C. De \& Labouriau L.G.. 1969. Corpos silicosos de gramíneas dos Cerrados. II. Pesquisa Agropecuária Brasileira, n.4, p. 143-151.

Cavalcante, P. B. 1968. Contribuição ao estudo dos corpos silicosos das gramíneas Amazônicas I. Panicoideae(Melinideae, Andropogoneae, e Tripsaceae). Botânica, 80:1-11.

Coe, H. H. G.; Alexandre, A.; Carvalho, C. N. de; Santos, G. M.; Silva, A. S.; Sousa, L. O. F.; Lepsch, I.F. 2012. Changes in Holocene tree cover density in Cabo Frio (Rio de Janeiro, Brazil): Evidence from soil phytolith assemblages. Quaternary International, n.2, p. 1-10.

Kealhofler, L.; Piperno, D. 1998.Opal Phytoliths in Southeast Asian Flora. Washington: Smithsonian Institution Press

Henderson, A. J.; Galeano, G.; Bernal, R. 1995. Field Guide to the palms of the americas. Princeton: Princeton University Press, p.352.

Lorenzi, H.; Nablick, L. R.; Kahn, F.; Ferreira, E. 2010.Flora Brasileira Lorenzi: Arecaceae (palmeiras). Nova Odessa: Instituto Plantarum, p.179.

Lorenzi, H. 1996. Palmeiras Brasileiras e Exóticas Cultivadas. Nova Odessa: Instituto Plantarum.

Lu, H. Y.; Wu, N. Q.; Liu, K. B.; Jiang, H.; Liu, T. S. 2007. Phytoliths as Quantitative Indicators for the Reconstruction of Past Environmental Conditions in China II: Palaeoenvironmental Reconstruction in the Loess Plateau. In Quaternary Science Reviews, n. 26(5), p. $759-772$.

Madella M., Alexandre; A. BALL; T. 2005. International Code for Phytolith Nomenclature 1.0, In Annals of Botany, n. 96(2), p. 253-260.

Madella, M. 2008. Physiology of silica deposition. Curso ministrado no 7th International Meeting on Phytolith Research, Mar del Plata.

Medeanic, S.; Dillenburg, S. R.; Weschenfelder, J. 2007. Palinomorfos nos sedimentos de fundo de laguna dos Patos, RS: Aplicação nas reconstruções paleoambientais. In Gravel, n. 5(1), p. 89-102.
Medeanic, S.; Cordazzo, C. V.; Corrêa, I. C. S.; Mirlean, N. 2008.Os Fitólitos em Gramíneas de Dunas do Extremo Sul do Brasil: Variabilidade Morfológica e Importância nas Reconstruções Paleoambientais Costeiras. In Gravel, n. 6(2), p. 1 - 14.

Monteiro, M. R.; Parolin, M.; Guerreiro, R. L.; Stevaux, J. C.; Pereira, J. S. R. Primeiras considerações paleoambientais com análise de fitólitos em sedimentos turfosos nos Campos Gerais do Estado do Paraná. In: XIII Congresso da associação brasileira de estudos do Quaternário III Encontro do Quaternário Sul-americano, 2011, Armação dos Búzios - RJ. O Quaternário Sul Americano: Desafios e Perspectivas, 2011.

Osterrieth, M. 2008. Rol e importancia del estudio de las biomineralizaciones en Biología,Geología y Arqueología. Curso ministrado no 7th Interantional Meeting on Phytolith Research, Mar del Plata.

Piperno, D.R. 1988. A phytolith analysis: an archaeological and geological perspective. Academic Press, London, p. 280.

Piperno, D. R. 2006. Phytolithis: a comprehensive guide for archaeologists andpaleoecologists. 1.ed. Oxford: AltaMira Press, p.238.

Raitz, E. 2012. Coleção de referência de silicofitólitos da flora do sudoeste do Paraná: subsídios para estudos paleoambientais. 153 f. Dissertação de Mestrado, Universidade Estadual do Oeste do Paraná, Francisco Beltrão, Paraná.

Rasbold, G. G.; Monteiro, M. R.; Parolin, M.; Caxambú, M. G. 2011. Formas de fitólitos presentes em Butiaparaguayensis (Barb. Rodr.) L. H. Bailey (Arecaceae) InII Simpósio Ambiental da Universidade Tecnológica Federal do Paraná. Campo Mourão, Paraná.

Runge, F. 1999. The opal phytolith inventory of soils in Central Africa - Quantities, shapes, classification, and spectra. Review of Paleobotany and Palynology, $\mathrm{n}$. 107 , p. 23-53.

Sendulsky, T. S.; Labouriau, L. G. (Ed.).1966. Corpos silicosos de gramíneas dos cerrados. In Anais do Simpósio Sobre o Cerrado. Academia Brasileira de Ciências 38, suplemento, p. 159-170. 
Zucol, A.F., Bertoldi de Pomar, H., Osterrieth, M. \& Brea, M. Bibliografía sobre análisis fitolíticos. GEFACS, v.1, 1999.

Zucol, A. F.; Brea, M.; Scopel, A. 2005.First record of fossil wood and phytolith assemblages of the Late Pleistocene in El Pal- mar National Park (Argentina).In Journal of South American Earth Sciences, n. 20, p. $33-43$.

Recebido em 02.VII.2012.

Aceito em 30.XI.2012. 